\title{
Desempeño productivo de novillos sobre pasturas templadas con suplementación energética en autoconsumo
}

\author{
Rovira, P.J. \\ Instituto Nacional de Investigación Agropecuaria (INIA), Ruta 8 km 281, CP 33000, Treinta y Tres, Uruguay. \\ Tel (598) 44521047, Fax (598)445 25701. E-mail: provira@tyt.inia.org.uy.
}

\begin{abstract}
Resumen
Rovira, P.J.: Desempeño productivo de novillos sobre pasturas templadas con suplementación energética en autoconsumo. Rev. vet. 23: 1, 3-7, 2012. La suplementación de bovinos en autoconsumo puede ser una alternativa válida para disminuir los costos de tiempo y mano de obra asociados al suministro diario de ración. El objetivo fue evaluar la suplementación energética en autoconsumo en el engorde de novillos ( $326 \pm 23 \mathrm{~kg}$ ) sobre pasturas templadas de alta calidad. Los tratamientos fueron: 1) testigo sin suplementación, 2) suplementación diaria $1 \%$ del peso vivo (PV), 3) suplementación restringida en autoconsumo $1 \% \mathrm{PV}$ con recarga semanal del comedero, 4) suplementación ad libitum en autoconsumo. En los tratamientos con autoconsumo se utilizó ración con $10 \%$ de sal para limitar consumo. El consumo de ración en autoconsumo ad-libitum fue $6,7 \mathrm{~kg} / \mathrm{a} / \mathrm{d}(1,8 \% \mathrm{PV})$, significativamente más alto que el registrado en los tratamientos al 1\% PV, ya sea bajo suministro diario $(3,6 \mathrm{~kg} / \mathrm{a} / \mathrm{d}) \mathrm{o}$ autoconsumo restringido $(3,7 \mathrm{~kg} / \mathrm{a} / \mathrm{d})$. En éste último tratamiento, el comedero quedó desprovisto de ración 2 a 3 días por semana para ajustar el nivel de suplementación a 1\% PV. El peso vivo final y la ganancia de peso fue significativamente mayor $(\mathrm{p}<0,05)$ en los animales suplementados ( $382 \pm 29 \mathrm{~kg}$ y $1,012 \pm 0,546 \mathrm{~kg} / \mathrm{a} / \mathrm{d}$, respectivamente) comparado con el grupo testigo ( $329 \pm 25 \mathrm{~kg}$ y $0,198 \pm 0,276 \mathrm{~kg} / \mathrm{a} / \mathrm{d})$, no existiendo diferencias asociadas al método de entrega de la ración $(p>0,05)$. La eficiencia de conversión ( $\mathrm{kg}$ de ración para ganar $1 \mathrm{~kg}$ de PV adicional comparado con el grupo testigo) tendió a desmejorar en los tratamientos de autoconsumo (5,2 y $6,9 \mathrm{~kg}$ restringido y ad-libitum, respectivamente) comparado con la suplementación diaria $(4,7 \mathrm{~kg})$. Los animales suplementados registraron mayor área de ojo de bife y grasa intramuscular y subcutánea que los animales sin suplementación. La suplementación en autoconsumo es una estrategia válida para facilitar operativamente esquemas de suplementación, aunque biológicamente la eficiencia de conversión puede verse afectada por el consumo de ración con alto contenido de sal.
\end{abstract}

Palabras claves: bovino, suplementación, autoconsumo, sal.

\begin{abstract}
Rovira, P.J.: Evaluation of self-feeding energetic supplementation on performance of steers grazing temperate pastures. Rev. vet. 23: 1, 3-7, 2012. Self-feeding of supplements to livestock can be a useful practice among those producers limited on time and labor resources to handle daily supplementation of cattle. The objective of the study was to evaluate self-feeding of an energetic supplement on the performance of steers (326 $\pm 23 \mathrm{~kg})$ grazing high quality temperate pastures. Treatments were: 1) control group without supplementation, 2) hand-fed daily supplementation at $1 \%$ of body weight (BW), 3) restricted self-feeding supplementation at $1 \%$ BW loading the feeder once a week, 4) self-feeding supplementation ad-libitum. The ration used in self-feeding treatments contained $10 \%$ salt to limit food intake. Feed intake in self-feeding ad-libitum supplementation $(6.7 \mathrm{~kg} / \mathrm{a} / \mathrm{d})$ was greater than that registered in supplemented treatments at $1 \% \mathrm{BW}$ either hand-fed $(3.6 \mathrm{~kg} / \mathrm{a} / \mathrm{d})$ or restricted self-feeding $(3.7 \mathrm{~kg} / \mathrm{a} / \mathrm{d})$. In this last treatment, the feeder was empty during 2 to 3 days per week to adjust feed intake at $1 \%$ BW. The final BW and average daily gain of supplemented animals $(382 \pm 29 \mathrm{~kg}$ and $1.012 \pm 0.546 \mathrm{~kg} / \mathrm{a} / \mathrm{d}$, respectively) were greater $(\mathrm{p}<0.05)$ compared to those obtained in the control group $(329 \pm 25 \mathrm{~kg}$ and $0.198 \pm 0.276 \mathrm{~kg} / \mathrm{a} / \mathrm{d})$, without differences associated with the delivery method of the ration $(p>0.05)$. Conversion efficiency of the supplement, expressed as the amount of supplement $(\mathrm{kg})$ required to gain $1 \mathrm{~kg}$ of live weight above the performance of the control group, tended to worsen in self-feeding treatments (5.2 and $6.9 \mathrm{~kg}$ restricted and ad-libitum, respectively) compared to the hand-fed daily
\end{abstract}

Recibido: 5 enero 2012 / Aceptado: 12 marzo 2012 


\begin{abstract}
supplementation $(4.7 \mathrm{~kg})$. Supplemented animals registered a greater rib eye area and fat (intramuscular and subcutaneous) than animals in the control group. Self-feeding supplementation is a useful strategy to cope with the high cost and/or lack of labor in livestock operations although supplement feed conversion can be negatively affected due to the high intake of ration and its associated salt.
\end{abstract}

Key words: cattle, supplementation, self-feeding, salt.

\section{INTRODUCCIÓN}

La producción de carne bovina en Uruguay tradicionalmente ha estado basada en sistemas pastoriles sobre campo natural. En los últimos años, la adopción de prácticas más intensivas de producción se ha expandido con el objetivo de incrementar la dotación y productividad animal ${ }^{7}$. Una de las alternativas de intensificación es la introducción de especies de gramíneas y/o leguminosas de mayor producción y valor nutritivo. Raigrás (Lolium multiflorum), trébol blanco (Trifolium repens) y Lotus sp son las opciones forrajeras más utilizadas en regiones de clima templado ${ }^{15,18,21}$. Condiciones climáticas durante el invierno determinan una disminución de la tasa de crecimiento de dichas especies disminuyendo la capacidad de carga del sistema e indicando la necesidad de suplementar los animales para mantener niveles elevados de productividad.

La suplementación implica un costo adicional, no sólo del suplemento en sí, sino también del recurso tiempo y mano de obra implicados en la rutina diaria de acarreo y traslado de la ración. Por tal motivo, los productores han comenzado a adoptar el sistema de autoconsumo de ración que no requiere la manipulación diaria del suplemento. Se basa en la utilización de comederos provistos con un depósito en donde a medida que los animales consumen el alimento disponible en la batea el re-abastecimiento es automático a través de la caída por gravedad de la ración desde el depósito (Figura 1). La principal desventaja es que se debe agregar cantidad importantes de sal $(\mathrm{NaCl})$ en la ración para limitar el consumo y evitar trastornos ruminales (acidosis). A nivel internacional, existen resultados contradictorios del efecto de la sal en el desempeño productivo de bovinos $6,8,11$.

El objetivo del presente trabajo fue evaluar el efecto de la suplementación energética en autoconsumo en el desempeño productivo de novillos sobre pasturas de alta calidad.

\section{MATERIAL Y MÉTODOS}

El experimento se desarrolló en el Instituto Nacional de Investigación Agropecuaria (INIA) en la localidad de Treinta y Tres, región Este del Uruguay (latitud $33^{\circ} 14^{\prime} \mathrm{S}$, longitud: $54^{\circ} 15^{\prime} \mathrm{O}$ ). El diseño experimental consistió en bloques completamente al azar con cuatro tratamientos sin repeticiones. Treinta y dos novillos de $1 \frac{1}{2}$ años Aberdeen Angus (peso inicial $\pm \mathrm{DE}=326 \pm$ $23 \mathrm{~kg}$ ) fueron asignados al azar en cuatro tratamientos
(8 novillos/tratamiento): 1) testigo sin suplementación, 2) suplementación diaria al $1 \%$ del peso vivo (PV), 3) suplementación restringida en autoconsumo al $1 \% \mathrm{PV}$, 4) suplementación ad libitum en autoconsumo.

La base forrajera fue una mezcla de $2^{\circ}$ año de Trifolium repens (trébol blanco), Lotus corniculatus (lotus) y Lolium multiflorum (raigrás). En cada tratamiento la dotación fue de 3 novillos/ha en sistema de pastoreo rotativo con cambios semanales de franja de pastoreo a una asignación diaria de forraje de 3,0\%. El período efectivo de suplementación fue de 63 días comprendidos entre el 24 de junio y el 26 de agosto de 2009 (no incluye periodo de acostumbramiento). El comedero de autoconsumo en el tratamiento restringido se recargó semanalmente cada lunes al 1\% PV x 7 días, en tanto en el tratamiento ad-libitum se recargó regularmente evitando que quedara desprovisto de ración.

La ración fue de origen comercial recomendada para engorde de novillos sobre pasturas de calidad y la composición fue la misma con la diferencia que en autoconsumo se incluyó $10 \%$ de sal $(\mathrm{NaCl})$ para limitar el consumo animal según recomendación del fabricante (la ración de suministro diario tenía $0,5 \% \mathrm{NaCl}$ ). $\mathrm{La}$ cantidad de ración suministrada en el tratamiento de autoconsumo restringido al $1 \% \mathrm{PV}$ se corrigió por el $10 \%$ de sal, de manera que la cantidad de nutrientes suministrados fuera comparable con el tratamiento suplementación diaria al $1 \% \mathrm{PV}$. El valor nutritivo de la ración de suministro diario fue $14,3 \%$ proteína cruda (PC), 7,5\% fibra detergente ácida (FDA) y 13,5\% cenizas (C); y para la ración de autoconsumo fue $10,5 \%$ PC; $5,1 \%$ FDA y $22,4 \% \mathrm{C}$.

La disponibilidad, altura y composición botánica del forraje se determinó cada 14 días mediante cortes al ras del suelo. Las muestras de materia seca (general) y de las fracciones gramínea y leguminosa se enviaron al Laboratorio de Nutrición Animal de INIA al inicio y fin del experimento para determinación de digesti-

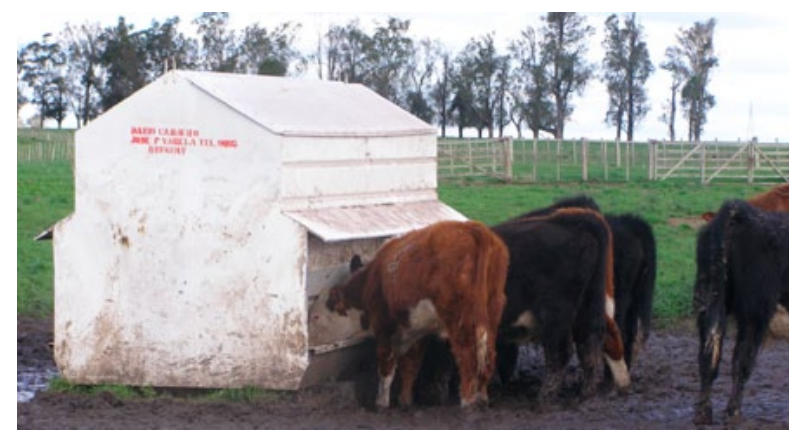

Figura 1. Comedero de autoconsumo. 
bilidad de la materia orgánica (DMO), proteína cruda (PC), fibra detergente neutro (FDN), fibra detergente ácida (FDA) y cenizas (C). Los animales se pesaron sin desbaste cada 21 días a primera hora de la mañana. Al inicio y final del experimento se registraron las variables de área de ojo de bife $(\mathrm{cm} 2)$ en el músculo longissimus dorsi, así como grasa intramuscular (\%) y espesor de grasa subcutánea $(\mathrm{mm})$ entre la $12^{\mathrm{a}}$ y $13^{\mathrm{a}}$ costilla mediante la técnica de ultrasonido.

Las variables fueron sometidas a un análisis de varianza utilizando un diseño completamente al azar sin repeticiones. Se utilizó el paquete estadístico SAS versión 9.12 (SAS Institute, Cary, NC, USA). El forraje disponible (MS $\mathrm{kg} / \mathrm{ha}$ ) y la altura del tapiz $(\mathrm{cm}) \mathrm{se}$ analizaron en forma independiente en cada fecha de muestreo y promediando sobre el periodo experimental, utilizando el comando PROC GLM. La ganancia diaria de peso individual ( $\mathrm{kg} / \mathrm{a} / \mathrm{día})$ fue estimada a través de la regresión lineal del peso vivo en el tiempo. El modelo estadístico para la variable peso vivo $(\mathrm{kg})$ fue de medidas repetidas de covarianzas autoregresivas e incluyó los factores fijos del tratamiento y fecha de pesada (período), y el efecto aleatorio del animal dentro de cada tratamiento utilizando el comando PROC MIXED. En todos los casos, las medias de los efectos fijos significativos $(p<0,05)$ se separaron mediante el comando LSMEANS. Los resultados para cada variable se presentan como media \pm desvío estándar (DE).

\section{RESULTADOS}

No existieron diferencias significativas en la disponibilidad y altura de forraje entre tratamientos $(p>$ $0,05)$. Medias y $\mathrm{DE}$, promediando sobre tratamientos durante el periodo experimental, fueron de $1.297 \pm$ $406 \mathrm{kgMS} /$ ha y $10,8 \pm 2,3 \mathrm{~cm}$, respectivamente. $\mathrm{Al}$ inicio del experimento la composición botánica, en base seca, fue $58 \%$ trébol blanco, $10 \%$ raigrás, $6 \%$ lotus y $26 \%$ otras especies (malezas, gramíneas, restos secos). El valor nutritivo del forraje disponible se incrementó al avanzar el periodo experimental expresado fundamentalmente a través de una mayor digestibilidad de la materia orgánica (60 y $75 \%$, respectivamente) y proteína cruda (19,7 y 23,7\%, respectivamente) (Figura 2). Comparando el valor nutritivo de las 2 principales especies introducidas en el tapiz, el raigrás presentó un menor nivel de proteína $(22,0 \%)$ y mayor contenido de fibra detergente neutro $(57,5 \%)$ comparado con el trébol blanco ( $25,7 \%$ y $40,5 \%$, respectivamente).

En el tratamiento suplementación ad libitum en autoconsumo los animales consumieron significativamente más ración $(6,7 \mathrm{~kg} / \mathrm{a} / \mathrm{d}$, equivalente al 1,8\% PV) que los animales suplementados al $1 \%$ del peso vivo ya sea diariamente $(3,6 \mathrm{~kg} / \mathrm{a} / \mathrm{d})$ o en autoconsumo restringido $(3,7 \mathrm{~kg} / \mathrm{a} / \mathrm{d})$. En este último tratamiento, el comedero quedaba desprovisto de ración entre el $4^{\circ}$ y $5^{\circ}$ día luego de haber realizado la recarga semanal, por lo que los animales quedaban entre 2 y 3 días alimentados únicamente a pasto ya que se trataba de un sistema de recarga del comedero a tiempo fijo para limitar el consumo de ración a $1 \%$ PV en caso de que la sal adicional en la ración no fuera lo suficientemente efectiva como limitador de consumo. El consumo estimado de $\mathrm{NaCl}$ proveniente de la ración fue de 18,370 y 670 g/a/día en los novillos suplementados diariamente, en autoconsumo restringido y ad-libitum, respectivamente.

El peso vivo final de los animales en los tratamientos suplementados $(382 \pm 29 \mathrm{~kg})$ fue mayor $(\mathrm{p}<0,05)$ que el peso vivo de los animales sin suplementación $(329 \pm 25 \mathrm{~kg})$ (Figura 3). No existieron diferencias en peso vivo final de los animales entre los tratamientos con suplementación $(p>0,05)$.

La ganancia diaria de peso vivo promedio $\pm \mathrm{DE}$ (kg/a/día) fue 0,198 $\pm 0,276$ (testigo), 0,960 $\pm 0,493$ (suministro diario $1 \% \mathrm{PV}$ ), $0,908 \pm 0,662$ (autoconsumo restringido $1 \% \mathrm{PV}$ ) y $1,166 \pm 0,506$ (autoconsumo ad libitum). La ganancia de peso de los animales promediando sobre los tratamientos suplementados $(1,012 \pm$ $0,546 \mathrm{~kg} / \mathrm{a} / \mathrm{d})$ fue mayor $(\mathrm{p}<0,05)$ que la ganancia de peso de los animales sin suplementación. No existieron diferencias en ganancia de peso de los animales entre los tratamientos con suplementación ( $p>0,05)$. La eficiencia de conversión, expresada como los kg de ración para ganar $1 \mathrm{~kg}$ de peso vivo adicional comparado con el desempeño productivo de los animales en el tratamiento testigo sin suplementación, fue de 4,7 (sumi-

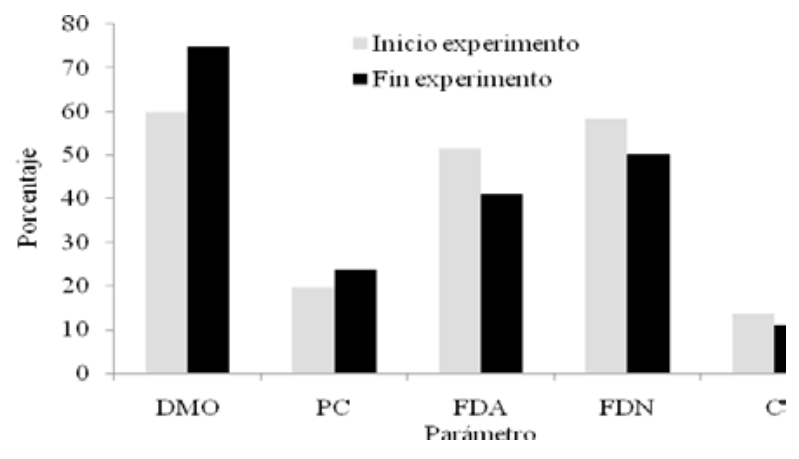

Figura 2. Valor nutritivo del forraje ofrecido al inicio y fin del experimento (DMO: digestibilidad materia orgánica, PC: proteína cruda, FDA: fibra detergente ácida, FDN: fibra detergente neutro, C: cenizas).

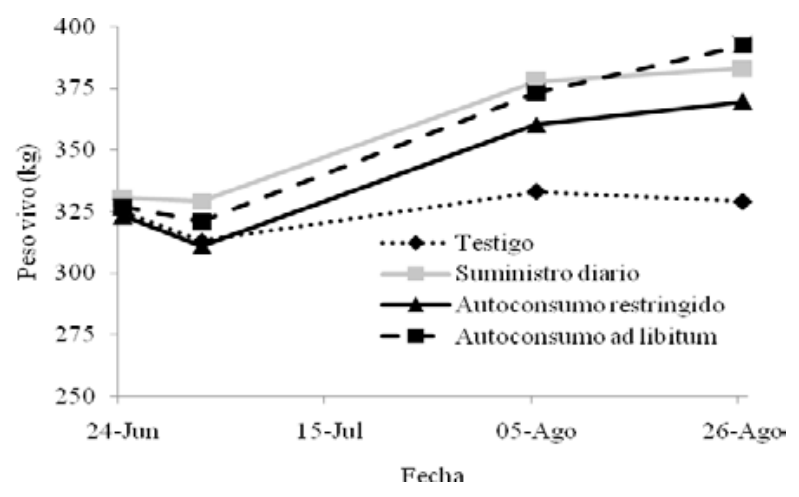

Figura 3. Evolución de peso vivo de los animales. 
Tabla 1. Registro de las variables de ultrasonido al final del experimento (media $\pm \mathrm{DE}$ ).

\begin{tabular}{lcccc}
\hline \multirow{2}{*}{ variable } & \multicolumn{4}{c}{ tratamiento } \\
\cline { 2 - 5 } & testigo & $\begin{array}{c}\text { suministro } \\
\text { diario }\end{array}$ & $\begin{array}{c}\text { autoconsumo } \\
\text { restringido }\end{array}$ & $\begin{array}{c}\text { autoconsumo } \\
\text { ad libitum }\end{array}$ \\
\hline \multirow{2}{*}{ AOB, cm cm $^{2}$} & $49,9^{\mathrm{a}}$ & $54,5^{\mathrm{a}}$ & $54,3^{\mathrm{a}}$ & $60,7^{\mathrm{b}}$ \\
& $\pm 5,38$ & $\pm 3,53$ & $\pm 3,18$ & $\pm 5,64$ \\
GIM, \% & $2,37^{\mathrm{a}}$ & $2,77^{\mathrm{a}}$ & $2,49^{\mathrm{a}}$ & $2,76^{\mathrm{a}}$ \\
& $\pm 0,48$ & $\pm 0,31$ & $\pm 0,22$ & $\pm 0,24$ \\
EGS, mm & $3,86^{\mathrm{a}}$ & $4,13^{\mathrm{a}}$ & $4,33^{\mathrm{a}}$ & $4,08^{\mathrm{a}}$ \\
& $\pm 0,47$ & $\pm 0,64$ & $\pm 0,81$ & $\pm 1,24$ \\
\hline
\end{tabular}

AOB: área ojo de bife, GIM: grasa intramuscular, EGS: espesor de grasa subcutánea. ${ }^{\text {abc }}$ Letras diferentes en una misma fila indican diferencias significativas $(p>0,05)$.

nistro diario 1\% PV), 5,2 (autoconsumo restringido 1\% PV) y 6,9 (autoconsumo ad libitum).

Los valores promedio $\pm \mathrm{DE}$ al inicio del trabajo experimental del área de ojo de bife (AOB), grasa intramuscular (GIM), y espesor de grasa subcutánea (EGS) fueron $46,6 \pm 3,73 \mathrm{~cm}^{2}, 2,48 \pm 0,45 \%, 2,94 \pm 0,72 \mathrm{~mm}$, respectivamente, no existiendo diferencias significativas entre tratamientos $(\mathrm{p}>0,05)$. Al final del periodo experimental, los animales suplementados en autoconsumo ad libitum registraron mayor AOB comparado con el resto de los tratamientos $(\mathrm{p}<0,05)$ (Tabla 1). Los animales suplementados al $1 \% \mathrm{PV}$ tendieron a registrar más AOB que los animales del tratamiento testigo ( $\mathrm{p}$ $=0,085)$. Las diferencias en las variables de grasa no llegaron a ser significativas $(\mathrm{p}>0,05)$.

\section{DISCUSIÓN}

La suplementación tuvo un efecto significativo en la ganancia de peso de los animales, independientemente del método de entrega de la ración. La ganancia diaria de peso fue 1,012 y $0,198 \mathrm{~kg} / \mathrm{a} / \mathrm{d}$ en novillos con y $\sin$ acceso a ración. El efecto positivo de la suplementación no sólo debe ser analizado en el corto plazo sino también en un contexto global de utilización de los recursos alimenticios. Una alta dotación durante el invierno, cuando la producción de forraje es mínima, permite incrementar la utilización del forraje durante la primavera siguiente, estación de máximo crecimiento de las pasturas ${ }^{14}$.

El nivel de inclusión de $10 \%$ de sal limitó el consumo de ración entre $1,7-1,8 \% \mathrm{PV}$ en los tratamientos de autoconsumo, equivalente a 5,8-6,8 kg/a/día. En bovinos adultos en pastoreo, trabajos publicados a nivel internacional sitúan el nivel de sal en la ración entre 20 y $30 \%$ para limitar el consumo del suplemente en un rango de $0,80-2,0 \mathrm{~kg} / \mathrm{a} / \mathrm{d}^{5,16,17}$. En ovinos, corderos suplementados con grano de cebada disminuyeron el consumo de 384 a $170 \mathrm{~g} / \mathrm{a} / \mathrm{d}$ al incrementar el contenido de sal de 10 a $30 \%{ }^{19}$. La alta tasa de consumo de ración registrada en el presente experimento determinó que el comedero en el tratamiento de autoconsumo restringido quedara desprovisto de ración entre 2 y 3 días por semana, equivalente a un $28-42 \%$ del tiempo, como forma de no superar el umbral de suplementación de $1 \% \mathrm{PV}$ definido para dicho tratamiento.

La suplementación diaria produjo una diferencia numérica 5,7\% superior en ganancia de peso comparado con la estrategia de autoconsumo restringido a un mismo nivel de suplementación (1\% PV). Dos razones pueden explicar dicha diferencia. En primer lugar, la ausencia de ración en el comedero de autoconsumo restringido durante determinados días, como se mencionó anteriormente, puede haber afectado el desempeño de los animales. En segundo lugar, el mayor consumo de sal en autoconsumo implica un mayor costo energético de mantenimiento en el animal asociado al metabolismo y excreción de la sal. Numerosos trabajos han cuantificado los cambios en la concentración de $\mathrm{Na}, \mathrm{Cl}$ y $\mathrm{K}$ en la sangre de animales alimentados con raciones con alto contenido de sal con diversos resultados $1,5,8,11,20$.

En la medida que haya suficiente agua de bebida disponible, la mayoría de la sal ingerida es excretada a través de la orina ${ }^{13}$. A pesar de la reducción numérica en la ganancia de peso de los animales en el tratamiento de autoconsumo restringido, la misma no fue significativa comparado con el suministro diario y el ahorro en tiempo y mano de obra por visitar el potrero una vez por semana en vez de ir todos los días justifica la técnica desde el punto de vista operativo. Técnicamente, no es recomendable que el comedero quede desprovisto de ración para asegurar un suministro uniforme de ración y un ambiente ruminal estabilizado.

La mayor ganancia de peso de los animales suplementados en autoconsumo ad-libitum se atribuyó a un consumo de ración significativamente mayor comparado con el consumo de los animales suplementados al $1 \%$ PV. Similares resultados fueron reportados en terneros sobre campo natural, en donde animales suplementados en autoconsumo con ración ( $10 \%$ de sal) registraron una ganancia de peso $34 \%$ superior comparado con terneros suplementados diariamente con la misma ración sin sal debido a un mayor consumo diario de ración en autoconsumo $(1,36 \% \mathrm{PV})$ comparado con el suministro diario $(1 \% \mathrm{PV})^{3}$.

La tasa diaria de consumo de sal en ganado bovino varía entre 0,05 y $0,15 \%$ PV cuando la sal es utilizada como limitador de consumo ${ }^{10}$. Considerando el peso vivo y consumo de ración promedio de los animales en el presente experimento, la tasa de consumo de sal registrada fue de 0,11 y $0,19 \% \mathrm{PV}$ en los tratamientos de autoconsumo restringido al $1 \%$ PV y ad-libitum, respectivamente. Existió un exceso de consumo de sal en los novillos suplementados en autoconsumo ad-libitum, más aún si se considera que el valor reportado puede estar subestimado para ciertos animales.

En sistemas de alimentación existe una alta variabilidad entre animales en el consumo de ración y en la tolerancia a la ingesta excesiva de sal, que la mayoría de las veces pasa desapercibida por la realización de cálculos promedio referidos al grupo de animales suplementados al no poder diferenciar consumo individual $^{4,9}$. Adicionalmente, el ganado bovino tiende a 
incrementar el consumo de sal con el paso del tiempo, por lo que la cantidad de sal en el suplemento debería ser ajustada periódicamente para mantener un consumo constante de ración ${ }^{2,4}$.

La eficiencia de conversión del suplemento a peso vivo tendió a desmejorar en los tratamientos de autoconsumo, especialmente cuando fue ad-libitum. La presencia de sal adicional y un mayor consumo de ración en los tratamientos de autoconsumo pueden haber explicado dicho incremento numérico en la eficiencia de conversión. Disminución de la digestibilidad de la materia orgánica, fibra y proteína, incremento de la excreción de nitrógeno y almidón en heces, y reducción de la actividad celulolítica a nivel ruminal, son algunos de los efectos reportados en la literatura internacional asociados al consumo de raciones con alto contenido de sal que pueden influir en la respuesta animal obtenida $5,6,12,13$.

En las variables registradas por ultrasonido, el área de ojo de bife (AOB) fue la más afectada a través de un incremento significativo de su valor en el tratamiento de autoconsumo ad-libitum asociado al mayor consumo de ración. Promediando sobre los tratamientos suplementados, la suplementación permitió incrementar los valores de AOB y grasa intramuscular un $13 \%$ y la grasa subcutánea un $8 \%$, comparado con los animales en el grupo testigo sin suplementación. De haberse extendido el período de suplementación es de esperar que se incremente la magnitud de la diferencia entre los animales con y sin acceso a ración en las variables de ultrasonido.

En conclusión, bajo las condiciones que se realizó el presente experimento, la suplementación energética en autoconsumo fue una estrategia válida para disminuir el tiempo y mano de obra incurridos en esquemas de suplementación diaria, sin afectar negativamente el desempeño de los animales. La tecnología de autoconsumo requiere una continua supervisión técnica para controlar el nivel de consumo de ración y los efectos negativos que un exceso de sal pueden tener en la eficiencia de conversión y salud de los animales.

\section{REFERENCIAS}

1. Amaral DM, Croom WJ, Rakes AH, Leonard ES, Linnerud AC. 1985. Increased concentration of sodium chloride on milk production of cows fed low fiber diets. $J$ Dairy Sci 68: 2940-2947.

2. Beeson WM, Perry TW, Mohler M. 1957. Self-feeding free choice vs self-feeding a complete mixture for fattening steers. J Anim Sci 16: 787-795.

3. Blasina M, Piñeyrúa A, Renau M. 2010. Evaluación del sistema de autoconsumo para la suplementación invernal de terneras sobre pasturas naturales. Tesis de Grado, Facultad de Agronomía, Universidad de la República, Montevideo, Uruguay.

4. Bowman JP, Sowell BF. 1997. Delivery method and supplement consumption by grazing ruminants: A review. $J$ Anim Sci 75: 543-550.
5. Chicco CF, Schultz TA, Rios J, Plasse D, Burguera M. 1971. Self-feeding salt-supplement to grazing steers under tropical conditions. J Anim Sci 33: 142-146.

6. Croom WJ, Harvey RH, Linnerud AC, Froetschel M. 1982. High levels of sodium chloride in beef cattle diets. Can J Anim Sci 62: 217-227.

7. Del Campo M, Brito G, Soares de Lima JM, Vaz Martins D, Sañudo C, San Julián R, Hernández P, Montossi F. 2008. Effects of feeding strategies including different proportion of pasture and concentrate, on carcass and meat quality traits in Uruguayan steers. Meat Sci 80: 753760.

8. Harvey RW, Croom WJ, Pond KR, Hogarth BW, Leonard ES. 1986. High levels of sodium chloride in supplements for growing cattle. Can J Anim Sci 66: 423-429.

9. Hentges JF, Adams JR, Moore JE, Oltjen RR. 1967. Control of beef cattle forage supplement intake. J Anim Sci 26 (Suppl.1): 208.

10. Kunkle WE, Johns JT, Poore MH, Herd DB. 2000. Designing supplementation programs for beef cattle fed forage-based diets. J Anim Sci 77: 1-11.

11. Meyer JH, Weir WC, Ittner NR, Smith JD. 1955. The influence of high sodium chloride intakes by fattening sheep and cattle. J Anim Sci 14: 412-418.

12. Moseley G, Jones DI. 1974. The effect of sodium chloride supplementation of a sodium adequate hay on digestion, production and mineral nutrition of sheep. J Agr Sci 83: 37.

13. Nelson AB, MacVicar RW, Archer W, Meiske JC. 1955. Effect of a high salt intake on the digestibility of ration constituents and on nitrogen, sodium, and chloride retention by steers and wethers. J Anim Sci 14: 825-830.

14. Rearte DH, Pieroni GA. 2001. Supplementation of temperate pastures. Proceed XIX Internat Grassland Congr (Sao Paulo, Brazil), p. 679-689.

15. Rebuffo M, Bemhaja M, Risso D. 2006. Utilization of forage legumes in pastoral systems: state of art in Uruguay. Lotus Newsletter 36: 22-33.

16. Riggs JK, Colby RW, Sells LV. 1953. The effects of selffeeding salt-cottonseed meal mixtures to beef cows. $J$ Anim Sci 12: 379-393.

17. Schauer CS, Lardy GP, Slanger WD, Bauer ML, Sedivec KK. 2004. Self-limiting supplements fed to cattle grazing native mixed-grass prairie in the northern Great Plains. J Anim Sci 82: 298-306.

18. Soca P, Ayala W, Bermúdez R. 2002. The effect of herbage allowance of Lotus pedunculatus cv. Grasslands Maku on winter and spring beef heifer performance. Proceed New Zealand Grassland Assoc 64: 81-84.

19. Villa M, Buratovich O, Ceballos D. 2007. Uso de sal común $(\mathrm{NaCl})$ como limitador del consumo de suplemento invernal en corderas. Rev Arg Prod Anim 27: 76-78.

20. Weir WC, Miller RF. 1953. The use of salt as a regulator of protein supplement intake by breeding ewes. J Anim Sci 12: 219-225.

21. Wen YY, Jiang HF. 2005. Cutting effects on growth characteristics, yield composition, and population relationships of perennial ryegrass and white clover in mixed pastures. New Zeal J Agr Res 48: 349-358. 